upskilling a member of school staff to support these children, whilst also identifying children who need specialised support with access to our trained counsellors.

Aims Targeting all children affected by grief and loss: providing education and support to school staff, the parents and families, alongside counselling and therapy programmes for pupils. Giving help closer to home, via a trusted school staff member, and helping kids keep some semblance of 'normality' in their lives, when everything else might feel as though it is falling apart. Training a link worker in every primary/secondary school with our service and offering guidance around bereavement and significant loss. Teachers are on the frontline when it comes to the emotional wellbeing of pupils, they are the crucial element in providing that stability for children struggling with loss.

Method Model of training a link worker with ongoing support from the coordinator, within each school is both cost effective but also upskilling staff.

Results Started with 46 schools, now 74, and plan to expand. Conclusion Providing teachers with extra skills they can steer a child through even the most challenging situations. Additional support from the schools link service has dramatically changed the way youngsters are helped. Many of these children would drop out of education for a time: with our schools link service, they stay in.

\section{0-7 THE IMPLEMENTATION OF PALLIATIVE AND END OF LIFE CARE STANDARDS IN SCOTTISH PRISONS}

Gail Allan. Macmillan, NHS Scotland, Scottish Prison Service

\subsection{6/bmjspcare-2017-hospice.7}

Background The Scottish Government's Strategic Framework for Action on Palliative and End of Life Care shares a vision where palliative and end of life care is available to all. With an ageing prison population, the Scottish Prison Service has to deal with more foreseeable deaths from terminal and incurable illnesses than ever before. This brings new challenges for both prison regimes and prison facilities in providing quality palliative and end of life care for those prisoners.

Aims This two-year project aims to implement palliative and end of life care standards within a prison context to ensure access to an equitable quality service.

Initial Aims of Project Introduction visits to meet with prison service and NHS staff to discuss role and introduce them to the standards of care. Objectives for each prison based on a gap analysis and identification of education and training requirements. Identify members of staff as palliative care champions. Work collaboratively with each prison to identify their palliative population.

Results All Scottish prisons have been visited and preliminary work of introduction completed with identification of key sites for implementation of tests of change. Delivered sessions on 'What is palliative and end of life care?' Developed Palliative and Supportive Care registers to support the identification of those who may benefit from palliative care. Support new multidisciplinary prison palliative care meetings. Delivery of Anticipatory Care Planning training to prison and NHS staff. Process mapping of palliative and end of life service provision in key establishment to bench mark against standards of care and evaluate further areas of change.

Next Steps
- Evaluate first year of project.

- Develop training for prisoners

- Develop an education and support platform for Palliative Care Prison champions.

- Focused engagement with other stakeholders i.e., specialist palliative care and third sector organisations.

\section{0-8 PROACTIVE COLLABORATION WITH LOCAL PRISON SERVICES}

Helen Knowles, Tina Turner. Wakefield Hospice, Wakefield, UK

10.1136/bmjspcare-2017-hospice.8

The Wakefield Hospice in common with many others, is looking to diversify its income generation strategies in order to bring in an even greater financial contribution, and to bridge the growing gap between statutory funding and the ongoing and increasing costs of service provision. In developing this collaborative project with HMPs New Hall and Wakefield, the aim is to bring about benefits for both organisations and therefore for their specific 'populations': providing useful occupation for the residents of both prisons, facilitating access to affordable clothing and improved self-esteem, and providing an opportunity for a vulnerable group to make a positive contribution to the community, specifically those requiring the care of the hospice teams. The project has begun with the establishment of a 'hospice shop' within HMP New Hall and staffed by residents, and there are plans to extend our collaborative relationship with the development of a furniture upcycling initiative at HMP Wakefield, and the production in both settings of a range of saleable items. Benefits for the hospice are clearly financial, with shop sales already in excess of those anticipated, and estimated to increase significantly when the furniture scheme is bedded in: relationships between the organisations are now excellent, and this venture has facilitated new and important links with a community group previously not in communication with the hospice. The intention is to continue to build on the early successes, and possibly use the developing source of saleable products to reach out to other hospices with an offer of sales expansion.

\section{Parallel session 3: Real world practice: referrals, access and partnerships}

\section{0-9 PRIORITISING REFERRALS TO A SPECIALIST PALLIATIVE CARE SERVICE}

Margaret Clifford, Carl Stanborough, Thomas Osborne. St Joseph's Hospice, London, UK

\subsection{6/bmjspcare-2017-hospice.9}

Introduction With increasing referrals to specialist palliative care (SPC) services at a time of limited resources, effective triage systems are essential. This community SPC service uses a 'RAG' triage system, whereby a multidisciplinary team (MDT) decides daily if referrals should be categorised as 'Red' (seen within 24 hours), 'Amber' (3 days), or 'Green' (10 days).

Aims To assess if the MDT correctly prioritises referrals according to palliative care need. 